\title{
IC 10: a starburst galaxy resolved into individual stars
}

\author{
Deidre A. Hunter \\ Lowell Observatory, \\ 1400 West Mars Hill Road, Flagstaff, AZ 86001, USA
}

\begin{abstract}
We present a preliminary analysis of HST images of the Local Group galaxy IC 10. IC 10 has an unusual Wolf-Rayet star population and is believed to be undergoing a starburst. Nevertheless, stars to at least $6.5 \mathrm{M}_{\odot}$ are seen and the young star clusters are comparable in richness and spatial concentration to LMC populous clusters.
\end{abstract}

The Local Group irregular galaxy IC 10 is the nearest example of the galaxywide starburst phenomenum. As such, it offers a unique opportunity to test ideas about the star formation process in such an environment. In particular it has been suggested that starburst galaxies, have unusually high lower stellar mass limits (up to 10-20 $\mathrm{M}_{\odot}$ ) and/or unusual stellar initial mass functions (IMFs). Therefore, we have obtained HST images of a portion of IC 10, and we present preliminary analysis of the F814W and F336W images.

We have constructed a luminosity function for all the stars measured in all four CCDs in the F814W filter and corrected for the underlying older galaxy. We measure stars to about $6.5 \mathrm{M}_{\odot}$. Thus, the lower stellar mass limit is $\leq 6.5 \mathrm{M}_{\odot}$. Although this upper limit is relatively high, the lower mass limit is less than the $10-20 \mathrm{M}_{\odot}$ that is suggested for some systems from global measurements and from theory.

We have identified six bright star clusters in the PC and two in the W4. They have diameters of 13-40 pc. The richness (number of stars) and spatial concentration (stars formed per unit area) of the clusters are comparable to the populous cluster NGC 1818 in the LMC or the knots of stars in Constellation III in the LMC. However, they are significantly less rich and less concentrated than the globular-like cluster R 136 in the 30 Doradus nebula in the LMC. Thus, the starburst in IC 10 has not produced super star clusters but rather more normal star-forming regions. This is also the case with the BCD galaxies IZw 18 and VII Zw 403 which we have examined with HST. Therefore, it is not reasonable to assume that a starburst containing giant $\mathrm{H}$ II regions has necessarily produced super star clusters.

Acknowledgments. Support for this work is provided by NASA through grant number GO-06406.01-95A. 